DOI: $10.5965 / 223811711622017102$

\title{
A eficácia do controle químico varia com o genótipo de azevém e a temperatura do ar no momento da aplicação
}

The efficiency of chemical control varies on the genotype of italian ryegrass and the air temperature at the time of spraying

\author{
Liese de Vargas Pereira ${ }^{1,2}$, Leonardo Bianco de Carvalho ${ }^{2,3^{*}}$ e Taísa Dal Magro ${ }^{4}$ \\ Recebido em 19/10/2016/ Aceito em 03/01/2017
}

\section{RESUMO}

Este trabalho visou estudar alternativas para o manejo químico de azevém (Lolium multiflorum), levando em consideração fatores ambientais e biológicos no momento da aplicação dos herbicidas, bem como a possível resposta diferencial entre biótipos da espécie. Objetivou-se avaliar a influência da temperatura do ar no controle químico de dois biótipos de azevém. $\mathrm{O}$ experimento foi conduzido em casa de vegetação, em delineamento inteiramente casualizado com quatro repetições e organizado em esquema fatorial $2 \times 2 \times 10$ (biótipo, temperatura e herbicida). Um biótipo foi proveniente de área com cultivos anuais e o outro, de cultivo perene. Os herbicidas testados foram: clethodim, clodinafop-propargyl, fenoxapropp-ethyl+clethodim, glyphosate, iodosulfuronmethyl, paraquat, paraquat+diuron, sethoxydim e tepraloxydim. A aplicação ocorreu em plantas mantidas sob temperatura do ar de $20-22{ }^{\circ} \mathrm{C}$ e $30-34{ }^{\circ} \mathrm{C}$. O controle foi avaliado aos $7,14,21$ e 28 dias após a aplicação dos tratamentos, atribuindo-se o percentual de 0 a 100 que correspondeu à ausência de injúria e morte das plantas, respectivamente. Os dados foram submetidos à análise de variância e as médias comparadas pelo teste de Tukey $(\mathrm{P}=0,05)$. Em geral, os herbicidas foram mais eficazes em temperatura de $20-22{ }^{\circ} \mathrm{C}$, sendo que a temperatura de $30-34{ }^{\circ} \mathrm{C}$ prejudicou o controle do azevém. A suscetibilidade aos graminicidas foi dependente do biótipo em aplicação sob temperatura de $30-34{ }^{\circ} \mathrm{C}$.

PALAVRAS-CHAVE: Lolium multiflorum, herbicidas, condição ambiental.

\section{ABSTRACT}

This work aimed to study alternatives to the chemical management of italian ryegrass (Lolium multiflorum), taking into account environmental and biological factors at the time of herbicide application, as well as the possible differential response among biotypes of the species. The objective of this study was to evaluate the influence of air temperature on the chemical control of two ryegrass biotypes. The experiment was conducted in a greenhouse, in a completely randomized design with four replicates and organized in a $2 \times 2 \times 10$ factorial scheme (biotype, temperature and herbicide). One biotype was obtained from an area with annual crops and the other from a perennial crop. The tested herbicides were: clethodim, clodinafop-propargyl, fenoxapropp-ethyl+clethodim, glyphosate, iodosulfuronmethyl, paraquat, paraquat+diuron, sethoxydim and tepraloxydim. The application occurred in plants maintained in air temperature of $20-22{ }^{\circ} \mathrm{C}$ and 30 $34{ }^{\circ} \mathrm{C}$. The control was evaluated at $7,14,21$ and 28 days after application of the treatments, assigning the percentage of 0 to 100 that corresponded to the absence of injury and death of the plants, respectively. The data was submitted to analysis of variance and the means were compared by the Tukey test $(\mathrm{P}=0.05)$. In general, herbicides were more effective at a temperature of $20-22{ }^{\circ} \mathrm{C}$, and the temperature of 30 $34{ }^{\circ} \mathrm{C}$ impaired ryegrass control. The susceptibility to the grass killers was dependent on the biotype in application under temperature of $30-34{ }^{\circ} \mathrm{C}$.

KEYWORDS: Lolium multiflorum, herbicides, environmental condition.

${ }^{1}$ Secretaria Estadual da Agricultura, Pecuária e Irrigação do Rio Grande do Sul, Porto Alegre, RS, Brasil.

${ }^{2}$ Universidade do Estado de Santa Catarina, Lages, SC, Brasil.

${ }^{3}$ Universidade Estadual Paulista, Jaboticabal, SP, Brasil.

${ }^{4}$ Universidade de Caxias do Sul, Vacaria, RS, Brasil.

*Autor para correspondência <lbcarvalho@fcav.unesp.br> 


\section{INTRODUÇÃO}

O azevém (Lolium multiflorum) é considerado uma planta daninha de grande importância econômica que afeta negativamente lavouras destinadas à produção de grãos no inverno, pomares e vinhedos da região Sul do Brasil (ROMAN et al. 2004). No sistema de plantio direto ou em pomares, a dessecação ou controle dessa espécie é realizado, normalmente, com a aplicação de herbicidas não seletivos, em diferentes estádios fenológicos, sendo o glyphosate o herbicida mais utilizado para esse fim (CHRISTOFFOLETI \& LÓPEZ-OVEJERO 2003).

A absorção dos herbicidas é um processo bifásico que consiste na rápida penetração pela cutícula, seguido pela absorção simplástica, sendo que a duração deste processo depende das características físico-químicas e dose do herbicida, das características estruturais próprias da espécie a ser controlada, do estádio de desenvolvimento e da biologia da planta daninha, bem como, as técnicas de aplicação e os fatores ambientais (PROCÓPIO et al. 2003). Entre os fatores ambientais, a temperatura, a umidade relativa do ar e o nível de irradiância afetam diversas características morfológicas e fisiológicas das plantas daninhas, incluindo composição e quantidade de substâncias lipofílicas depositadas sobre a superfície foliar, ângulo foliar, taxa fotossintética entre outras, os quais determinarão maior ou menor permeabilidade da cutícula das plantas e, em consequência disto, na quantidade do produto absorvido (VIDAL 2002).

A temperatura e a umidade relativa do ar, por exemplo, influenciam também no tempo de permanência da gotícula pulverizada, no aumento da hidratação da cutícula e na abertura estomática, podendo aumentar ou diminuir o transporte de solutos na planta. Altas temperaturas podem melhorar a absorção, por provocar maior fluidez dos lipídios da camada cuticular e da membrana celular. Todavia, também podem apresentar efeitos negativos devido à maior rapidez do secamento da gota pulverizada, provocando a cristalização do herbicida na superfície foliar (SILVA \& SILVA 2007).

Além dos fatores climáticos, os fatores biológicos da planta influenciam na absorção e translocação dos herbicidas. Entre os fatores biológicos, um dos mais importantes é o estádio fenológico da planta. Segundo CRAWFORD \& ZAMBRYSKI (2001), os tecidos mais novos possuem a capacidade de transportar moléculas maiores, o que torna as plantas em estádio mais avançados menos suscetíveis aos herbicidas.

Com isso, o objetivo do experimento foi avaliar a influência da temperatura do ar no controle químico de dois biótipos de azevém utilizando herbicidas de diferentes mecanismos de ação.

\section{MATERIAL E MÉTODOS}

O experimento foi conduzido em casa de vegetação, utilizando sementes de um biótipo de azevém coletado em área de cultivo anual (cereais) e outro, em área de cultivo perene (pomar). As sementes dos dois biótipos foram semeadas em bandejas plásticas e, ao atingirem o estádio de duas folhas, as plantas foram transplantadas para vasos com capacidade de $3 \mathrm{~L}$, com posterior manutenção de uma planta por vaso.

O delineamento experimental foi inteiramente casualizado com quatro repetições e os tratamentos foram agrupados em três fatores, constituindo um esquema fatorial $2 \times 2 \times 10$ :

- Fator A: Biótipo - Biótipo 1 (cultivo anual) e Biótipo 2 (cultivo perene);

- Fator B: Temperatura 1 de $20-22{ }^{\circ} \mathrm{C}$ e Temperatura 2 de $30-34{ }^{\circ} \mathrm{C}$

- Fator C: Herbicida - glyphosate (Roundup $\mathrm{WG}^{\circledR}$ - 2,5 kg ha-1); tepraloxydim $\left(\right.$ Aramo $^{\circledR}-0,5 \mathrm{~L} \mathrm{ha}^{-1}+$ $5 \mathrm{~mL}$ de Assist ${ }^{\circledR}$ ); iodosulfuron-methyl (Hussar ${ }^{\circledR}$ $\left.70 \mathrm{~g} \mathrm{ha}^{-1}\right)$; paraquat+diuron $\left(\right.$ Gramocil $^{\circledR}-2,0 \mathrm{~L}^{-} \mathrm{ha}^{-}$ $\left.{ }^{1}\right)$; paraquat $\left(\right.$ Gramoxone $^{\circledR}-2,0 \mathrm{~L} \mathrm{ha}^{-1}$ ); clodinafoppropargyl (Topik ${ }^{\circledR}$ - 0,25 L ha-1); fenoxaprop-petyl+clethodim $\left(\right.$ Selefen $\left.^{\circledR}-1,0 \mathrm{~L} \mathrm{ha}^{-1}\right)$; clethodim $\left(\right.$ Select $^{\circledR}-0,45 \mathrm{~L} \mathrm{ha}^{-1}+5 \mathrm{~mL}$ de Lanzar $\left.{ }^{\circledR}\right)$; sethoxydim $\left(\right.$ Poast $^{\circledR}-1,25 \mathrm{~L} \mathrm{ha}^{-1}$ ) e testemunha (sem aplicação de herbicida).'

A aplicação dos herbicidas foi realizada com pulverizador costal pressurizado a $\mathrm{CO}_{2}$, com pontas de pulverização do tipo leque, calibrado para vazão de $150 \mathrm{~L} \mathrm{ha}^{-1}$ de calda herbicida, aplicados em azevém nos estádios de 3-4 folhas, sob temperaturas do ar de $20-22{ }^{\circ} \mathrm{C}$ e $30-34{ }^{\circ} \mathrm{C}$ durante a aplicação. As plantas cresceram inicialmente em casas de vegetação separadas, mantidas sob as temperaturas supracitadas até o momento da aplicação. Após a aplicação, as plantas foram então mantidas nas mesmas condições de temperatura.

Avaliou-se o controle de azevém pelos herbicidas (\% de eficácia) aos 7, 14, 21 e 28 dias após o tratamento (DAT), utilizando-se de escala percentual 
na qual zero e cem, correspondem à ausência de injúria e morte das plantas, respectivamente.

Os dados foram submetidos à análise de variância (teste F) e as médias foram comparadas pelo teste de Tukey, a 5\% de probabilidade de erro.

\section{RESULTADOS E DISCUSSÃO}

Após a análise estatística dos dados, observouse que houve interação entre os três fatores testados, independente da data de avaliação.

Ao avaliar o efeito de herbicida no controle de azevém, observou-se que nas duas primeiras datas de avaliação (7 e 14 DAT), os herbicidas paraquat e paraquat+diuron proporcionaram os maiores índices de controle, independente do biótipo e temperatura no momento da aplicação (Tabela 1). Aos 14 DAT, de maneira geral, os herbicidas inibidores de ACCase, clethodim, clodinafop-propargyl, fenoxapropp-ethyl+clethodim, sethoxydim e tepraloxydim, atingiram níveis de controle acima de $90 \%$ para os dois biótipos quando aplicados à temperatura de 20$22{ }^{\circ} \mathrm{C}$, entretanto quando aplicados em temperatura mais elevada, observou-se redução dos índices de controle para alguns desses produtos. Os herbicidas clethodim e clodinafop-propargyl tiveram sua eficácia reduzida, independente do biótipo, enquanto que fenoxaprop-p-ethyl+clethodim e sethoxydim tiveram a eficácia reduzida no controle do biótipo resistente quando aplicados sob temperatura de $30-34{ }^{\circ} \mathrm{C}$. Dentre os graminicidas, apenas o herbicida tepraloxydim mostrou-se eficiente no controle dos dois biótipos, independente da temperatura de aplicação.

VARGAS \& ROMAN (2006) verificaram que, aos 7 DAT, paraquat apresentou índice de controle de azevém resistente ao glyphosate superior a $90 \%$. O controle eficiente, visualizado ainda nas primeiras avaliações, pode ser explicado pelo modo de ação desses herbicidas, que, por serem de contato, manifestam os sintomas logo após a aplicação, possibilitando o controle efetivo das plantas daninhas em um curto espaço de tempo, quando comparados aos herbicidas sistêmicos que possuem ação mais lenta (RODRIGUES \& ALMEIDA 2005).

Aos 21 DAT (Tabela 2), seguindo a tendência das avaliações anteriores, níveis de controle

Tabela 1 - Efeito da temperatura do ar no controle (\%) de dois biótipos (B1 - cultivo anual; B2 - cultivo perene) de Lolium multiflorum aos 7 e 14 dias após o tratamento (DAT) com herbicidas.

Table 1 - Effect of air temperature on the control (\%) of two biotypes (B1 - annual crop; B2 - perennial crop) of Lolium multiflorum at 7 and 14 days after treatment (DAT) using herbicides

\begin{tabular}{|c|c|c|c|c|c|}
\hline \multirow{2}{*}{ Herbicida } & \multirow{2}{*}{$\mathrm{T}^{1}$} & \multicolumn{2}{|c|}{07 DAT } & \multicolumn{2}{|c|}{ 14 DAT } \\
\hline & & B1 & B2 & B1 & B2 \\
\hline \multirow{2}{*}{ Clethodim } & $\mathrm{T} 1$ & $35 \mathrm{~d}$ & $43 \mathrm{~d}$ & $98 \mathrm{a}$ & $95 \mathrm{a}$ \\
\hline & $\mathrm{T} 2$ & $28 \mathrm{c}$ & $44 \mathrm{c}$ & $34 \mathrm{~d}$ & $54 c$ \\
\hline \multirow{2}{*}{ Clodinafop-propargyl } & $\mathrm{T} 1$ & $30 \mathrm{~d}$ & $27 \mathrm{e}$ & $75 \mathrm{~b}$ & $95 \mathrm{a}$ \\
\hline & $\mathrm{T} 2$ & $30 \mathrm{c}$ & $16 \mathrm{deb}$ & $39 \mathrm{~cd}$ & $33 \mathrm{~d}$ \\
\hline \multirow{2}{*}{$\begin{array}{l}\text { Fenoxaprop-p- } \\
\text { ethyl+clethodim }\end{array}$} & $\mathrm{T} 1$ & $29 \mathrm{~d}$ & $26 \mathrm{~d}$ & $94 \mathrm{a}$ & $99 \mathrm{a}$ \\
\hline & $\mathrm{T} 2$ & $31 \mathrm{c}$ & $29 \mathrm{~d}$ & $50 \mathrm{bc}$ & $89 \mathrm{ab}$ \\
\hline \multirow{2}{*}{ Glyphosate } & $\mathrm{T} 1$ & $25 \mathrm{c}$ & $79 \mathrm{bc}$ & $99 \mathrm{a}$ & $99 \mathrm{a}$ \\
\hline & $\mathrm{T} 2$ & $13 \mathrm{~d}$ & $46 c$ & $18 \mathrm{f}$ & $79 \mathrm{~b}$ \\
\hline \multirow{2}{*}{ Iodosulfuron-methyl } & $\mathrm{T} 1$ & $68 \mathrm{~b}$ & $75 \mathrm{bc}$ & $78 \mathrm{~b}$ & $78 \mathrm{~b}$ \\
\hline & $\mathrm{T} 2$ & $24 \mathrm{~cd}$ & $25 \mathrm{de}$ & $33 \mathrm{e}$ & $33 \mathrm{e}$ \\
\hline \multirow{2}{*}{ Paraquat } & $\mathrm{T} 1$ & $99 \mathrm{a}$ & $98 \mathrm{a}$ & $99 \mathrm{a}$ & $99 \mathrm{a}$ \\
\hline & $\mathrm{T} 2$ & $97 \mathrm{a}$ & $99 \mathrm{a}$ & $99 a$ & $99 a$ \\
\hline \multirow{2}{*}{ Paraquat+diuron } & $\mathrm{T} 1$ & $99 \mathrm{a}$ & $99 \mathrm{a}$ & $99 \mathrm{a}$ & $99 \mathrm{a}$ \\
\hline & $\mathrm{T} 2$ & $98 \mathrm{a}$ & $99 a$ & $99 a$ & $99 a$ \\
\hline \multirow{2}{*}{ Sethoxydim } & $\mathrm{T} 1$ & $73 \mathrm{~b}$ & $86 \mathrm{~b}$ & $99 \mathrm{a}$ & $94 \mathrm{a}$ \\
\hline & $\mathrm{T} 2$ & $29 \mathrm{~cd}$ & $43 \mathrm{c}$ & $60 \mathrm{~b}$ & $98 \mathrm{a}$ \\
\hline \multirow{2}{*}{ Tepraloxydim } & $\mathrm{T} 1$ & $69 \mathrm{~b}$ & $70 \mathrm{ac}$ & $98 \mathrm{a}$ & $97 \mathrm{a}$ \\
\hline & $\mathrm{T} 2$ & $80 \mathrm{~b}$ & $75 \mathrm{~b}$ & $96 \mathrm{a}$ & $99 \mathrm{a}$ \\
\hline \multirow{2}{*}{ Testemunha } & $\mathrm{T} 1$ & $00 \mathrm{e}$ & $00 \mathrm{f}$ & $00 \mathrm{c}$ & $00 \mathrm{c}$ \\
\hline & $\mathrm{T} 2$ & $00 \mathrm{e}$ & $00 \mathrm{f}$ & $00 \mathrm{f}$ & $00 \mathrm{e}$ \\
\hline $\mathrm{CV} \%$ & & \multicolumn{2}{|c|}{12,45} & \multicolumn{2}{|c|}{8,7} \\
\hline
\end{tabular}

${ }^{1}$ Temperatura no momento da aplicação $\left({ }^{\circ} \mathrm{C}\right)$, T1 $\left(20-22{ }^{\circ} \mathrm{C}\right), \mathrm{T} 2\left(30-34{ }^{\circ} \mathrm{C}\right) \cdot{ }^{*}$ Médias seguidas pela mesma letra na coluna não diferem entre si, utilizando o teste de Tukey $(\mathrm{p} \leq 0,05)$. 
Tabela 2 - Efeito da temperatura do ar no controle (\%) de dois biótipos (B1 - cultivo anual; B2 - cultivo perene) de Lolium multiflorum aos 21 e 28 dias após o tratamento (DAT) com herbicidas.

Table 2 - Effect of air temperature on the control (\%) of two biotypes (B1 - annual crop; B2 - perennial crop) of Lolium multiflorum at 21 and 28 days after treatment (DAT) using herbicides.

\begin{tabular}{|c|c|c|c|c|c|}
\hline \multirow{2}{*}{ Herbicida } & \multirow[b]{2}{*}{$\mathrm{T}^{1}$} & \multicolumn{2}{|c|}{$21 \mathrm{DAT}$} & \multicolumn{2}{|c|}{$28 \mathrm{DAT}$} \\
\hline & & $\mathrm{B} 1$ & $\mathrm{~B} 2$ & $\mathrm{~B} 1$ & B2 \\
\hline \multirow{2}{*}{ Clethodim } & T1 & $99 \mathrm{a}$ & $97 \mathrm{a}$ & $99 \mathrm{a}$ & $89 \mathrm{a}$ \\
\hline & $\mathrm{T} 2$ & $43 \mathrm{~d}$ & $60 \mathrm{e}$ & $78 \mathrm{bc}$ & $99 \mathrm{a}$ \\
\hline \multirow{2}{*}{ Clodinafop- propargyl } & $\mathrm{T} 1$ & $93 \mathrm{a}$ & $75 \mathrm{c}$ & $99 \mathrm{a}$ & $89 \mathrm{a}$ \\
\hline & $\mathrm{T} 2$ & $66 \mathrm{bc}$ & $87 \mathrm{bc}$ & $78 \mathrm{bc}$ & $99 \mathrm{a}$ \\
\hline \multirow{2}{*}{$\begin{array}{l}\text { Fenoxaprop-p- } \\
\text { ethyl+clethodim }\end{array}$} & $\mathrm{T} 1$ & $99 \mathrm{a}$ & $99 \mathrm{a}$ & $99 \mathrm{a}$ & $99 \mathrm{a}$ \\
\hline & $\mathrm{T} 2$ & $63 \mathrm{c}$ & $83 \mathrm{~cd}$ & 74 bcd & $99 \mathrm{a}$ \\
\hline \multirow{2}{*}{ Glyphosate } & $\mathrm{T} 1$ & $99 \mathrm{a}$ & $99 \mathrm{a}$ & $99 \mathrm{a}$ & $99 \mathrm{a}$ \\
\hline & $\mathrm{T} 2$ & $24 \mathrm{e}$ & $75 \mathrm{~d}$ & $25 \mathrm{e}$ & $84 \mathrm{~b}$ \\
\hline \multirow{2}{*}{ Iodosulfuron-methyl } & $\mathrm{T} 1$ & $92 \mathrm{a}$ & $85 \mathrm{bc}$ & $98 \mathrm{a}$ & $96 \mathrm{a}$ \\
\hline & $\mathrm{T} 2$ & $77 \mathrm{~b}$ & $86 \mathrm{bcd}$ & $84 b$ & $94 \mathrm{ab}$ \\
\hline \multirow{2}{*}{ Paraquat } & $\mathrm{T} 1$ & $99 \mathrm{a}$ & $99 \mathrm{a}$ & $99 \mathrm{a}$ & $99 \mathrm{a}$ \\
\hline & $\mathrm{T} 2$ & $99 \mathrm{a}$ & $99 \mathrm{a}$ & 99 a & 99 a \\
\hline \multirow{2}{*}{ Paraquat+diuron } & $\mathrm{T} 1$ & $99 \mathrm{a}$ & $99 \mathrm{a}$ & $99 \mathrm{a}$ & $99 \mathrm{a}$ \\
\hline & $\mathrm{T} 2$ & $99 a$ & $97 \mathrm{a}$ & $99 a$ & $96 \mathrm{a}$ \\
\hline \multirow{2}{*}{ Sethoxydim } & $\mathrm{T} 1$ & $99 \mathrm{a}$ & $99 \mathrm{a}$ & $99 \mathrm{a}$ & $99 \mathrm{a}$ \\
\hline & $\mathrm{T} 2$ & $66 \mathrm{bc}$ & $99 a$ & $68 \mathrm{~cd}$ & $99 \mathrm{a}$ \\
\hline \multirow{2}{*}{ Tepraloxydim } & $\mathrm{T} 1$ & $99 \mathrm{a}$ & $99 \mathrm{a}$ & $99 \mathrm{a}$ & $99 \mathrm{a}$ \\
\hline & $\mathrm{T} 2$ & $98 \mathrm{a}$ & $91 \mathrm{abc}$ & $99 \mathrm{a}$ & $93 \mathrm{a}$ \\
\hline \multirow{2}{*}{ Testemunha } & $\mathrm{T} 1$ & $00 \mathrm{~b}$ & $00 \mathrm{~d}$ & $00 \mathrm{~b}$ & $00 \mathrm{c}$ \\
\hline & $\mathrm{T} 2$ & $00 \mathrm{f}$ & $00 \mathrm{f}$ & $00 \mathrm{e}$ & $00 \mathrm{c}$ \\
\hline $\mathrm{CV} \%$ & & \multicolumn{2}{|c|}{7,7} & \multicolumn{2}{|c|}{6,4} \\
\hline
\end{tabular}

${ }^{1}$ Temperatura no momento da aplicação $\left({ }^{\circ} \mathrm{C}\right), \mathrm{T} 1\left(20-22^{\circ} \mathrm{C}\right), \mathrm{T} 2\left(30-34{ }^{\circ} \mathrm{C}\right) .{ }^{*}$ Médias seguidas pela mesma letra na coluna não diferem entre si, utilizando o teste de Tukey $(\mathrm{p} \leq 0,05)$.

próximos a $100 \%$ foram obtidos com os herbicidas paraquat e paraquat+diuron, independente do biótipo e temperatura de aplicação. Os herbicidas clethodim, clodinafop-propargyl e fenoxaprop-pethyl+clethodim proporcionaram índices de controle acima de $90 \%$ para ambos os biótipos quando aplicados sob temperatura de $20-22{ }^{\circ} \mathrm{C}$, entretanto, com a aplicação dos herbicidas sob temperatura mais elevada, entre os graminicidas, somente tepraloxydim proporcionou controle satisfatório independente da temperatura de aplicação. Na última avaliação, aos 28 DAT (Tabela 2), de uma forma geral, a maioria dos herbicidas proporcionou índices de controle acima de $80 \%$ para o biótipo suscetível, independente da temperatura de aplicação. Níveis de controle abaixo de $80 \%$ foram observados somente no biótipo resistente para os herbicidas clethodim, clodinafop-propargyl, fenoxaprop-p-etyl+clethodim e sethoxydim quando aplicados em temperatura de $30-34{ }^{\circ} \mathrm{C}$; entretanto, na mesma temperatura, o biótipo suscetível foi totalmente controlado por esses herbicidas. Com relação à influência do biótipo no controle proporcionado pelos herbicidas, observou-se que não houve efeito deste fator no controle de azevém quando se utilizaram os herbicidas paraquat e paraquat + diuron, independente da data de avaliação.

No verão, com o decorrer do dia, a radiação global e a temperatura do ar se elevam e a umidade relativa do ar diminui (SKUTERUD et al. 1998, CIESLIK 2012). NASCIMENTO et al. (2012) observaram que a deposição das gotículas de herbicidas foram maiores no horário da manhã com temperatura de $18{ }^{\circ} \mathrm{C}$ em comparação ao horário da tarde com temperatura de $34^{\circ} \mathrm{C}$, quando as condições de temperatura e umidade relativa do ar são menos favoráveis às aplicações em pulverização.

Segundo ROMAN et al. (2007), os herbicidas inibidores de ACCase não são voláteis, entretanto a maioria degrada-se rapidamente na presença de luz quando ficam na superfície da folha, o que reduz sua eficácia. Baseando-se na literatura, pode-se inferir que, neste trabalho, a fotodegradação, a menor deposição, o menor tempo de permanência das gotas sobre as folhas e por consequência a menor absorção podem ter prejudicado a eficácia desses herbicidas quando aplicados sob temperatura do ar mais elevada. 
Por outro lado, a temperatura mais amena e a maior umidade relativa do ar permitiram que as gotas permanecessem sobre as folhas por um período mais longo, assim ocorreu a maior absorção dos produtos, o que explica a maior eficácia dos herbicidas aplicados na temperatura de $20-22^{\circ} \mathrm{C}$.

$\mathrm{Na}$ Tabela 3, verifica-se o efeito do biótipo em relação ao controle químico. Aos 7 DAT houve efeito de biótipo para os herbicidas glyphosate, clethodim e sethoxydim, os quais se mostraram mais eficazes no controle do biótipo suscetível, independentemente da temperatura de aplicação. O herbicida iodosulfuronmethyl também apresentou maior eficácia no controle do biótipo suscetível, entretanto somente quando aplicado na temperatura de $20-22{ }^{\circ} \mathrm{C}$. Na primeira data de avaliação, clodinafop-propargyl foi o único herbicida que mostrou maior eficiência no controle do biótipo resistente, se comparado com o suscetível, quando aplicado em temperatura de $30-34{ }^{\circ} \mathrm{C}$. Aos 14 DAT, houve efeito de biótipo para os herbicidas clethodim, fenoxaprop-p-etyl+clethodim, glyphosate, sethoxydim, clethodim e clodinafop-propargyl, os quais tiveram melhor ação no biótipo suscetível quando aplicados sob temperatura do ar mais elevada; entretanto não ocorreu este efeito quando os mesmos herbicidas foram aplicados sob temperatura de 20$22{ }^{\circ} \mathrm{C}$, sendo que os dois biótipos foram igualmente controlados.

Aos 21 DAT, todos os herbicidas graminicidas e também iodosulfuron-methyl foram mais eficientes no controle do biótipo suscetível quando aplicados na temperatura de $30-34{ }^{\circ} \mathrm{C}$, e essa tendência manteve-se até a última avaliação (Tabela 4). Aos 28 DAT, com a aplicação dos herbicidas em temperatura de 30 $34{ }^{\circ} \mathrm{C}$, à exceção de tepraloxydim, os graminicidas mantiveram índices de controle do biótipo resistente abaixo de $80 \%$ até a última data de avaliação, índice considerado ineficaz a campo, pois, segundo SOSBAI (2014), para ser considerado eficiente um herbicida precisa atingir índices de controle superiores a 90\%.

De maneira geral, não foi observado efeito de biótipo quando os herbicidas inibidores de ACCase foram aplicados na temperatura de $20-22{ }^{\circ} \mathrm{C}$, sendo que os mesmos controlaram igualmente o biótipo suscetível e o resistente ao glyphosate. ROMAN et al. (2004) relatam que os herbicidas inibidores de

Tabela 3 - Efeito do biótipo no controle (\%) de dois biótipos (B1 - cultivo anual; B2 - cultivo perene) de Lolium multiflorum aos 7 e 14 dias após o tratamento (DAT) com herbicidas.

Table 3 - Effect of the biotype on the control (\%) of two biotypes (B1 - annual crop; B2 - perennial crop) of Lolium multiflorum at 7 and 14 days after treatment (dat) using herbicides.

\begin{tabular}{|c|c|c|c|c|c|}
\hline \multirow{2}{*}{ Herbicidas } & \multirow[b]{2}{*}{$\mathrm{T}^{1}$} & \multicolumn{2}{|c|}{7 DAT } & \multicolumn{2}{|c|}{14 DAT } \\
\hline & & B1 & B2 & B1 & $\mathrm{B} 2$ \\
\hline \multirow{2}{*}{ Clethodim } & T1 & $35^{*}$ & 43 & $98^{\mathrm{ns}}$ & 95 \\
\hline & $\mathrm{T} 2$ & $28^{*}$ & 44 & $34^{*}$ & 54 \\
\hline \multirow{2}{*}{ Clodinafop-propargyl } & $\mathrm{T} 1$ & $30^{\text {ns }}$ & 27 & $75^{*}$ & 95 \\
\hline & $\mathrm{T} 2$ & $30^{*}$ & 16 & $39^{\text {ns }}$ & 33 \\
\hline \multirow{2}{*}{ Fenoxaprop-p-ethyl+clethodim } & $\mathrm{T} 1$ & $29^{\text {ns }}$ & 26 & $94^{\mathrm{ns}}$ & 99 \\
\hline & $\mathrm{T} 2$ & $31^{\text {ns }}$ & 29 & $50^{*}$ & 89 \\
\hline \multirow{2}{*}{ Glyphosate } & $\mathrm{T} 1$ & $25^{*}$ & 79 & $99^{\text {ns }}$ & 99 \\
\hline & $\mathrm{T} 2$ & $13^{*}$ & 46 & $18^{*}$ & 79 \\
\hline \multirow{2}{*}{ Iodosulfuron-methyl } & $\mathrm{T} 1$ & $68^{*}$ & 75 & $78^{\text {ns }}$ & 78 \\
\hline & $\mathrm{T} 2$ & $24^{\mathrm{ns}}$ & 25 & $33^{\text {ns }}$ & 33 \\
\hline \multirow{2}{*}{ Paraquat } & $\mathrm{T} 1$ & $99^{\text {ns }}$ & 98 & $99^{\mathrm{ns}}$ & 99 \\
\hline & $\mathrm{T} 2$ & $97^{\text {ns }}$ & 99 & $99^{\text {ns }}$ & 99 \\
\hline \multirow{2}{*}{ Paraquat+diuron } & $\mathrm{T} 1$ & $99^{\text {ns }}$ & 99 & $99^{\text {ns }}$ & 99 \\
\hline & $\mathrm{T} 2$ & $98^{\mathrm{ns}}$ & 99 & $99^{\text {ns }}$ & 99 \\
\hline \multirow{2}{*}{ Sethoxydim } & $\mathrm{T} 1$ & $73^{*}$ & 86 & $99^{\text {ns }}$ & 94 \\
\hline & $\mathrm{T} 2$ & $29^{*}$ & 43 & $60^{*}$ & 98 \\
\hline \multirow{2}{*}{ Tepraloxydim } & $\mathrm{T} 1$ & $69^{\text {ns }}$ & 70 & $98^{\mathrm{ns}}$ & 97 \\
\hline & $\mathrm{T} 2$ & $80^{\text {ns }}$ & 75 & $96^{\mathrm{ns}}$ & 99 \\
\hline \multirow{2}{*}{ Testemunha } & $\mathrm{T} 1$ & $00^{\mathrm{ns}}$ & 00 & $00^{\mathrm{ns}}$ & 00 \\
\hline & $\mathrm{T} 2$ & $00^{\text {ns }}$ & 00 & $00^{\text {ns }}$ & 00 \\
\hline $\mathrm{CV} \%$ & & \multicolumn{2}{|c|}{12,45} & \multicolumn{2}{|c|}{8,7} \\
\hline
\end{tabular}

${ }^{1}$ Temperatura no momento da aplicação $\left({ }^{\circ} \mathrm{C}\right), \mathrm{T} 1\left(20-22^{\circ} \mathrm{C}\right), \mathrm{T} 2\left(30-34{ }^{\circ} \mathrm{C}\right) .{ }^{*}$ Significativo, ${ }^{\mathrm{ns}}$ não significativo, utilizando teste de Tukey $(\mathrm{p} \leq 0,05)$. 
Tabela 4 - Efeito do biótipo no controle (\%) de dois biótipos (B1 - cultivo anual; B2 - cultivo perene) de Lolium multiflorum aos 21 e 28 dias após o tratamento (DAT) com herbicidas.

Table 4 -Effect of the biotype on the control (\%) of two biotypes (B1 - annual crop; B2 - perennial crop) of Lolium multiflorum at 21 and 28 days after treatment (dat) using herbicides.

\begin{tabular}{|c|c|c|c|c|c|}
\hline \multirow{2}{*}{ Herbicidas } & \multicolumn{3}{|c|}{$21 \mathrm{DAT}$} & \multicolumn{2}{|c|}{28 DAT } \\
\hline & $\mathrm{T}$ & B1 & $\mathrm{B} 2$ & B1 & B2 \\
\hline \multirow{2}{*}{ Cletodim } & $\mathrm{T} 1$ & $99^{\mathrm{ns}}$ & 97 & $99^{\text {ns }}$ & 89 \\
\hline & $\mathrm{T} 2$ & $43^{*}$ & 60 & $78^{*}$ & 99 \\
\hline \multirow{2}{*}{ Clodinafop-propargyl } & $\mathrm{T} 1$ & $93^{*}$ & 75 & $99^{\text {ns }}$ & 89 \\
\hline & $\mathrm{T} 2$ & $66^{*}$ & 87 & $78^{*}$ & 99 \\
\hline \multirow{2}{*}{ Fenoxaprop-p-ethyl+clethodim } & $\mathrm{T} 1$ & $99^{\text {ns }}$ & 99 & $99^{\text {ns }}$ & 99 \\
\hline & $\mathrm{T} 2$ & $63^{*}$ & 83 & $74^{*}$ & 99 \\
\hline \multirow{2}{*}{ Glyphosate } & $\mathrm{T} 1$ & $99^{\text {ns }}$ & 99 & $99^{\mathrm{ns}}$ & 99 \\
\hline & $\mathrm{T} 2$ & $24^{*}$ & 75 & $25^{*}$ & 84 \\
\hline \multirow{2}{*}{ Iodosulfuron-methyl } & $\mathrm{T} 1$ & $92^{\mathrm{ns}}$ & 85 & $98^{\text {ns }}$ & 96 \\
\hline & $\mathrm{T} 2$ & $77^{*}$ & 86 & $84^{*}$ & 94 \\
\hline \multirow{2}{*}{ Paraquat } & $\mathrm{T} 1$ & $99^{\text {ns }}$ & 99 & $99^{\text {ns }}$ & 99 \\
\hline & $\mathrm{T} 2$ & $99^{\text {ns }}$ & 99 & $99^{\text {ns }}$ & 99 \\
\hline \multirow{2}{*}{ Paraquat+diuron } & $\mathrm{T} 1$ & $99^{\text {ns }}$ & 99 & $99^{\text {ns }}$ & 99 \\
\hline & $\mathrm{T} 2$ & $99^{\mathrm{ns}}$ & 97 & $99^{\text {ns }}$ & 96 \\
\hline \multirow{2}{*}{ Sethoxydim } & $\mathrm{T} 1$ & $99^{\mathrm{ns}}$ & 99 & $99^{\text {ns }}$ & 99 \\
\hline & $\mathrm{T} 2$ & $66^{*}$ & 99 & $68^{*}$ & 99 \\
\hline \multirow{2}{*}{ Tepraloxydim } & $\mathrm{T} 1$ & $99^{\text {ns }}$ & 99 & $99^{\text {ns }}$ & 99 \\
\hline & $\mathrm{T} 2$ & $98^{\text {ns }}$ & 91 & $99^{\text {ns }}$ & 93 \\
\hline \multirow{2}{*}{ Testemunha } & $\mathrm{T} 1$ & $00^{\text {ns }}$ & 00 & $00^{\text {ns }}$ & 00 \\
\hline & $\mathrm{T} 2$ & $00^{\mathrm{ns}}$ & 00 & $00^{\text {ns }}$ & 00 \\
\hline $\mathrm{CV} \%$ & & \multicolumn{2}{|c|}{7,7} & \multicolumn{2}{|c|}{6,4} \\
\hline
\end{tabular}

${ }^{1}$ Temperatura no momento da aplicação $\left({ }^{\circ} \mathrm{C}\right), \mathrm{T} 1\left(20-22{ }^{\circ} \mathrm{C}\right), \mathrm{T} 2\left(30-34{ }^{\circ} \mathrm{C}\right) \cdot{ }^{*}$ Significativo, ${ }^{\text {ns }}$ não significativo, utilizando o teste de Tukey $(\mathrm{p} \leq 0,05)$.

ACCase, haloxyfop-re diclofop, controlamigualmente o biótipo sensível e o resistente ao glyphosate, quando aplicados no estádio de três a quatro folhas; entretanto, em geral, o tempo necessário para ocorrer a morte do biótipo resistente é maior do que aquele requerido para o biótipo sensível. Segundo FERREIRA et al. (2006), o biótipo suscetível e o resistente absorvem glyphosate na mesma intensidade; e HEREDIA et al. (1998) não observou diferenças marcantes na quantidade e na composição de cera epicuticular de biótipos de azevém resistente e suscetivel ao glyphosate, que segundo o autor é uma característica que define a maior absorção. BASTIANI et al. (2012) afirmam que a diferença dos níveis de controle dos biótipos de azevém aos herbicidas é mais evidente aos 7 DAT, sendo que o biótipo resistente ao glyphosate apresenta maior tolerância aos herbicidas testados nesta época.

A absorção reduzida dos herbicidas pelos biótipos em aplicações sob temperatura do ar elevada, aliada ao mecanismo que confere a resistência ao biótipo resistente, que consiste na reduzida translocação, são fatores que podem estar relacionados com a baixa eficácia dos herbicidas sistêmicos no controle do biótipo resistente observado neste trabalho. Resposta diferencial na absorção, na translocação e no metabolismo do herbicida amicarbazone, por exemplo, com dependência da temperatura do ar, foi observada em Poa annua, sendo que altas temperaturas reduzem a absorção e a translocação, mas aumentam o metabolismo do herbicida (YU et al. 2015). Efeitos equivalentes foram observados para seletividade de rimsulfuron em milho (KOEPPE et al. 2000) e bispyribac-sodium em Poa annua (MCCULLOUGH et al. 2009). Porém, maior absorção de herbicidas pode ser verificada em temperaturas mais altas (FLETCHER et al. 2007, MCCULLOUGH et al. 2010, 2013).

Com base nesses resultados, na utilização desses herbicidas como alternativas para o controle químico de azevém resistente em estádios iniciais deve ser dada atenção especial à temperatura do ar no momento de aplicação, devendo ser evitadas temperaturas acima de $30{ }^{\circ} \mathrm{C}$. 


\section{CONCLUSÃO}

A temperatura do ar no momento da aplicação afeta a eficácia do controle químico de azevém. Porém, a suscetibilidade do azevém a certos herbicidas é dependente do biótipo.

Os herbicidas paraquat e paraquat+diuron são mais eficazes no controle de azevém, independentemente do biótipo e da temperatura no momento da aplicação.

Quando aplicados a $20-22{ }^{\circ} \mathrm{C}$ de temperatura ambiente, os herbicidas são mais eficazes no controle de azevém, independente do biótipo.

Herbicidas aplicados sob a temperatura ambiente de $30-34{ }^{\circ} \mathrm{C}$ apresentam redução na eficácia de controle de azevém, com resposta diferencial entre biótipos.

\section{AGRADECIMENTOS}

Os autores agradecem ao CNPq pela concessão de bolsa de produtividade em pesquisa ao segundo autor.

\section{REFERÊNCIAS}

BASTIANI MO et al. 2012. Alternativas para o controle de azevém resistente ao glyphosate na fronteira oeste do Rio Grande do Sul. Campo Grande: XXVIII CBCPD. (CDRom).

CHRISTOFFOLETI PJ \& LÓPEZ-OVEJERO R. 2003. Principais aspectos da resistência de plantas daninhas ao herbicida glyphosate. Planta Daninha 21: 507-515.

CIESLIKLF. 2012. Interferência de fatores morfoambientais e horários de aplicação de fluazifop-p-butyl e fomesafen no controle de infestantes, seletividade e lucratividade da cultura do feijoeiro (Phaseolus vulgaris L.). Dissertação (Mestrado em Produção Vegetal). Pato Branco: UTFPR. $171 \mathrm{p}$.

CRAWFORD KM\& ZAMBRYSKIPC. 2001. Non-targeted and targeted protein movement through plasmodesmata in leaves in different developmental and physiological states. Plant Physiology 125: 1802-1812.

FERREIRA EA et al. 2006. Translocação do glyphosate em biótipos de azevém (Lolium multiflorum). Planta Daninha 24: 365-370.

FLETCHER AL et al. 2007. Transpiration responses to vapor pressure deficit in well watered 'slow-wilting' and commercial soybean. Environment Experimental Botany 61: 145-151.

HEREDIA A et al. 1998. La cutícula vegetal: estructura y funciones. Ecologia 12: 293-305.

KOEPPE MK et al. 2000. Basis of selectivity of the herbicide rimsulfuron in maize. Pest Biochemistry and Physiology 66: 170-181.

MCCULLOUGH PE et al. 2009. Bispyribac-sodium metabolism in annual bluegrass, creeping bentgrass, and perennial ryegrass. Weed Science 57: 470-473.

MCCULLOUGH PE et al. 2010. Amicarbazone efficacy on annual bluegrass and safety on cool-season turfgrasses. Weed Technology 24: 461-470.

MCCULLOUGH PE et al. 2013. Selectivity of methiozolin for annual bluegrass (Poa апnиа) control in creeping bentgrass as influenced by temperature and application timing. Weed Science 61: 209-216.

NASCIMENTO AB et al. 2012. Deposição de glifosato e utilização de adjuvante para diferentes pontas de pulverização e horários de aplicação. Pesquisa Aplicada e Agrotecnologia 5: 105-110.

PROCÓPIO SO et al. 2003. Estudos anatômicos de folhas de espécies de plantas daninhas de grande ocorrência no Brasil. III - Galinsoga parviflora, Crotalaria incana, Conyza bonariensis e Ipomoea cairica. Planta Daninha 21: 1-9.

RODRIGUES BN \& ALMEIDA FS. 2005. Guia de herbicidas. 5.ed. Londrina: IAPAR. 591p.

ROMAN ES et al. 2004. Resistência de azevém (Lolium multiflorum) ao herbicida glyphosate. Planta Daninha 22: 301-306.

ROMAN ES et al. 2007. Como funcionam os herbicidas: da biologia à aplicação. Passo fundo: Berthier. 160p.

SILVA AS \& SILVA JF. 2007. Tópicos em manejo de plantas daninhas. Viçosa: UFV. 367p.

SKUTERUD R et al. 1998. Effect of herbicides applied at different times of the day. Crop Protection 17: 41-46.

SOSBAI - Sociedade Sul-Brasileira de arroz irrigado. 2014. Arroz irrigado: recomendações técnicas da pesquisa para o Sul do Brasil. Santa Maria: SOSBAI. 192p.

VARGAS L \& ROMAN ES. 2006. Características e manejo de azevém resistente ao glyphosate. Passo Fundo: Embrapa Trigo. 9p. (Documentos online 59). Disponível em: https://ainfo.cnptia.embrapa.br/digital/bitstream/ CNPT-2010/40677/1/p-do59.pdf. Acesso em 19 out. 2016. VIDAL RA. 2002. Ação dos herbicidas: Absorção, translocação e metabolização. Porto Alegre: Evangraf. 89p. YU J et al. 2015. Physiological effects of temperature on turfgrass tolerance to amicarbazone. Pest Management Science 71: 571-578. 